ANNUAL PROGRESS REPORT (1991-1992)

Department of Energy Grant No. DE-FG02-84ER45130

Title: "Multi-Body Forces and The Energetics of Transition Metals, Alloys, and Semiconductors"

Principal Investigator:

A. E. Carlsson

Department of Physics

Washington University

St. Louis, Missouri 63130

\title{
DISCLAIMER
}

This report was prepared as an account of work sponsored by an agency of the United States Government. Neither the United States Gevernment nor any agency thereof, nor any of their employees, makes any warranty, express or implied, or assumes any legal liability or responsibility for the accuracy, completeness, or usefu!ness of any information, apparatus, product, or process disclosed, or represents that its use would not infringe privately owned rights. Reference herein to any specific commercial product, process, or service by trade name, trademark, manufacturer, or otherwise does not necessarily constitute or imply its endorsement, recommendation, or favoring by the United States Government or any agency thereof. The views and opinions of authors expressed herein do not necessarily state or reflect those of the United States Government or any agency thereof. 


\section{RESEARCH PROGRESS}

The progress over the past year basically divides into three areas:

(1) Potential-Energy Functions for Transition-Metal Aluminides

(Papers 1, 2, and 3).

We have developed a pair potential for transition-metal aluminides which is based on simply immersing two transition-metal impurities in a free-electron gas, and calculating the one-electron energy. The Hamiltonian is sufficiently simple that it can be solved exactly using Green's-function techniques; the parameters in the Hamiltonian were obtained from fits to $a b$-initio calculations for single impurities within a supercell approximation. The resulting pair potentials display an oscillating bchavior with large amplitude; the oscillations have a phase shift that depends on the $\mathrm{d}$-band filling. They are applicable only to cases in which transition metal atoms are not nearest neighbors. The potentials are remarkably accurate for transition metals to the right of $\mathrm{Ti}$ in the periodic table, agreeing to within $10-20 \%$ with ab-initio results for the $\mathrm{DO}_{22}$ vs. $\mathrm{Ll}_{2}$ energy differences. Since the structural-energy differences are closely related to antiphase-boundary energies, potentials of this type may be useful additions to embedded-atom type total-energy formulations. As yet, it is not possible to treat the Al-Ti system with the potentials; we hope, however, over the next year to make some progress in this direction. Paper 3 is a response to a published Comment by Linus Pauling regarding the relative importance of atomic-size effects and electronic terms in stabilizing the $\mathrm{Al}_{12} \mathrm{~W}$ structure, in which the importance of electronic terms of an angular nature is argued.

\section{(2) Electronic Structure and Energetics of Complex Structures and Quasicrystals (Papers 4, 5, 6 and 7).}

The aim of this work has been to understand the origins and existence criteria for quasigaps around the Fermi level in these materials. Such quasigaps appear to be strongly associated with structural stability, and also lead to remarkable transport properties. We have demonstrated that in free-electron materials, an important effect in quasigap formation is the topology of the "pseudo-Jones zone", a geometrical construct formed by bisecting the lines from the origin in reciprocal space to the dominant scattering vectors. We used a set of twelve scattering vectors which can smoothly interpolate between a small-unit cell cubic structure and an icosahedral structure. For physical values of the electron-ion pseudopotential, it is impossible for the cubic structure to have a gap in the density of states, because of degeneracy effects occurring th the edges of the pseudo-Jones zone. However, the icosahedral structure can have a gap, and we have performed numerical calculations which show that a gap can in fact develop if the pseuriopotential is sufficiently strong. These effects may be related to the formation of icosahedral phases and complex structures with icosahedral motifs at characteristic electron-per-atom ratios. Paper 6, on $\mathrm{Al}-\mathrm{Mn}$, is a write-up of work that was performed during the previous grant period; it showed that the Al-Mn quasicrystal has no overall quasigap in the DOS, but individual site-ptojected DOS distributions do. Paper 7, an invited paper for New's and Views in Nature, examines transport properties in some very high-quality stable ternary quasicrystals. 
(3) The remaining two papers, 8 and 9, deal with ceramic materials and fracture, respectively. Paper 8 extends the study of electron-interaction effects on band gaps that was begun some time ago to $\mathrm{PdO}$ and $\mathrm{PtO}$. In contrast with $\mathrm{NiO}$, a band gap of magnitude comparable to the experimental ones are obtained already at the level of the local-density approximation, although of course the interaction effects may still be important. This gap is caused by the reduced (tetragonal) symmetry of the structures, and probably contributes in turn to their stability. Paper 9 describes the beginning of an effort to establish the connection between the interatomic forces in solids and their fracture behavior. 


\section{RESEAR.CH PROGRESS}

The progress over the past year basically divides into three areas:

(1) Potential-Energy Functions for Transition-Metal Aluminides

(Papers 1, 2, and 3).

We have developed a pair potential for transition-metal aluminides which is based on simply immersing two transition-metal impurities in a free-electron gas, and calculating the one-electron energy. The Hamiltonian is sufficiently simple that it can be solved exactly using Green's-function techniques; the parameters in the Hamiltonian were obtained from fits to $a b$-initio calculations for single impurities within a supercell approximation. The resulting pair potentials display an oscillating behavior with large amplitude; the oscillations have a phase shift that depends on the $\mathrm{d}$-band filling. They are applicable only to cases in which transition metal atoms are not nearest neighbors. The potentials are remarkably accurate for transition metals to the right of $\mathrm{Ti}$ in the periodic table, agreeing to within $10-20 \%$ with ab-initio results for the $\mathrm{DO}_{22}$ vs. $\mathrm{Ll}_{2}$ energy differences. Since the structural-energy differences are closely related to antiphase-boundary energies, potentials of this type may be useful additions to embedded-atom type total-energy formulations. As yet, it is not possible to treat the Al-Ti system with the potentials; we hope, however, over the next year to make some progress in this direction. Paper 3 is a response to a published Comment by Linus Pauling regarding the relative importance of atomic-size effects and electronic terms in stabilizing the $\mathrm{Al}_{12} \mathrm{~W}$ structure, in which the importance of electronic terms of an angular nature is argued.

\section{(2) Electronic Structure and Energetics of Complex Structures and Quasicrystals (Papers 4, 5, 6 and 7).}

The aim of this work has been to understand the origins and existence criteria for quasigaps around the Fermi level in these materials. Such quasigaps appear to be strongly associated with structural stability, and also lead to remarkable transport properties. We have demonstrated that in free-electron materials, an important effect in quasigap formation is the topology of the "pseudo-Jones zone", a geometrical construct formed by bisecting the lines from the origin in reciprocal space to the dominant scattering vectors. We used a set of twelve scattering vectors which can smoothly interpolate between a small-unit cell cubic structure and an icosahedral structure. For physical values of the electron-ion pseudopotential, it is impossible for the cubic structure to have a gap in the density of states, because of degeneracy effects occurring at the edges of the pseudo-Jones zone. However, the icosahedral structure can have a gap, and we have performed numerical calculations which show that a gap can in fact develop if the pseudopotential is sufficiently strong. These effects may be related to the formation of icosahedral phases and complex structures with icosahedral motifs at characteristic electron-per-atom ratios. Paper 6, on $\mathrm{Al}-\mathrm{Mn}$, is a write-up of work that was performed during the previous grant period; it showed that the Al-Mn quasicrystal has no overall quasigap in the DOS, but individual site-projected DOS distributions do. Paper 7, an invited paper for News and Views in Nature, examines transport properties in some very high-quality stable ternary quasicrystals. 
(3) The remaining two papers, 8 and 9, deal with ceramic materials and fracture, respectively. Paper 8 extends the study of electron-interaction effects on band gaps that was begun some time ago to $\mathrm{PdO}$ and $\mathrm{PtO}$. In contrast with $\mathrm{NiO}$, a band gap of magnitude comparable to the experimental ones are obtained already at the level of the local-density approximation, although of course the interaction effects may still be important. This gap is caused by the reduced (tetragonal) symmetry of the structures, and probably contributes in turn to their stability. Paper 9 describes the beginning of an effort to establish the connection between the interatomic forces in solids and their fracture behavior. 


\section{PROPOSED RESEARCH}

(1) Methodology of obtaining potential-energy functions.

We intend to improve the aluminide potentials developed above to allow them to treat Group III and Group IV transition metals, in particular Ti. This will necessitate a better treatment of $\mathrm{p}-\mathrm{d}$ covalent bonding, and perhaps three-body transition-metal interactions as well. As first step, we will include the Al pseudopotential perturbatively. At the level of first-order perturbation theory, this can be done by evaluating the charge-density change due to the transitionmetal atoms, and integrating this with the pseudopotential. Three-body terms can also be evaluated by straightforward extension of the Green's-function methods, in which one simply obtains a larger scattering matrix than in the twobody case. For elemental transition metals, we intend to extend the methodology that was developed in the preceding grant period to transition metals with non-half-filled bands. This will probably necessitate the inclusion of odd moments of the electronic DOS; the coefficients of the terms will be obtained via scaling arguments from those of the even-moment terms, so that it will hopefully not be necessary to include extra fitting parameters. It may then be possible to treat $\mathrm{Rh}$ and Ir, whose negative Cauchy discrepancy renders the application of embedded-atom type method problematic.

(2) Applications of potential-energy functions.

The angular-force method for elemental bcc transition metals developed under the preceding grant period will be applied to grain boundaries, and to the development of a model Hamiltonian for the W(100) surface. The grainboundary work is being carried on in collaboration with Professor Vitek's group at the University of Pennsylvania, and involves comparison of results obtained by methods having a large range of speeds and accuracies. The surface work builds on the earlier calculations which showed that our angular-force method can treat the surface reconstruction fairly well. During the past year, we have developed simple surface-reconstruction criteria based on the forces, which are found to correspond fairly closely to ab-initio results. We hope to be able to turn these into a simple model Hamiltonian which could be used, for example, to elucidate the nature of the high-temperature unreconstructed phase. In aluminides, we intend to apply the pair potentials that have been developed to the stability of complex structures. The aim will be to find out whether or not the stable structures tend to have interatomic separations which correspond to minima of the pair potential. 


\section{STATEMENT OF OUTSIDE RESEARCH SUPPORT}

Funding outside of DOE Grant No. DE-FG02-ER-45130:

Office of Naval Research

"Lattice Green's Function Calculations of Crack-Tip Processes via QuantumMechanical Force Laws", part of coordinated set of proposals on mechanics and atomistics of mechanical behavior, led by Drs. Anthony Evans and James Rice.

$\$ 63,659$, per year to begin September 1,1992

Grant contains no summer support for Principal Investigator 


\section{PAPERS DURING DOE FUNDING PERIOD \\ (Pre/Reprints Enclosed)}

1. "Constant Volume" Pair Potential for Al-Transition Metal Compounds

J. Zou and A. E. Carlsson

Phys. Rev. B (submitted)

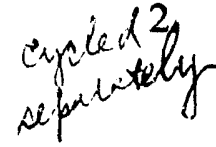

Phase Stability and Electronic Structure of Transition-Metal Aluminides

A. E. Carlsson

Proceedings of International Conference on the Physics of Transition Metals, Darmstadt, 1992 (in press)

3. Reply to "Comment on 'Relative Stability of the $\mathrm{Al}_{12} \mathrm{~W}$ Structure in Al-Transition-Metal Compounds"

A. E. Carlsson

Phys. Rev. B 45, 7511 (1992)

4. The Icosahedral Plane-Wave Model: Band-Gap Creation and Band-Tail States

A. E. Carlsson

J. Non-Cryst. Solids (in press)

5. Band Gap Creation by Icosahedral Symmetry in Nearly-Free-Electron Materials

A. E. Carlsson

Phys. Rev. B (submitted)

6. Electronic Structure of Quasicrystalline Al-Mn

H. Deng and A. E. Carlsson

Phys. Rev. Letters (submitted)

7. And Now Quasisemiconductors?"

A. E.Carlsson

Nature 353, 15 (1991)

8. Band Structures of Non-Magnetic Transition-metal Oxides: PdO and PtO

K. C. Hass and A. E. Carlsson

Phys. Rev. B (Brief Report) (in press)

9. Lattice Imperfections by Lattice Green's Functions

R. Thomson, S. J. Zhou, A. E. Carlsson, and V. K. Tewary

Phys. Rev. B (submitted) 
INVITED TALKS AND PAPERS DURING GRANT PERIOD

1. "Angular Forces and Structural Energetics: Transition Metals and Al-Transition Metal Compounds"

rot International Workshop on Developing Potentials for Atomistic attached, Simulations (September 1991, Ann Arbor)

2. "And Now Quasisemiconductors?"

Solicited article for Nature 353, 15 (1991).

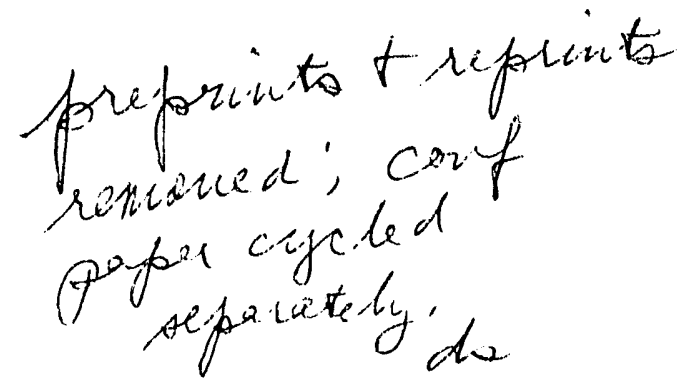

$-7-$ 

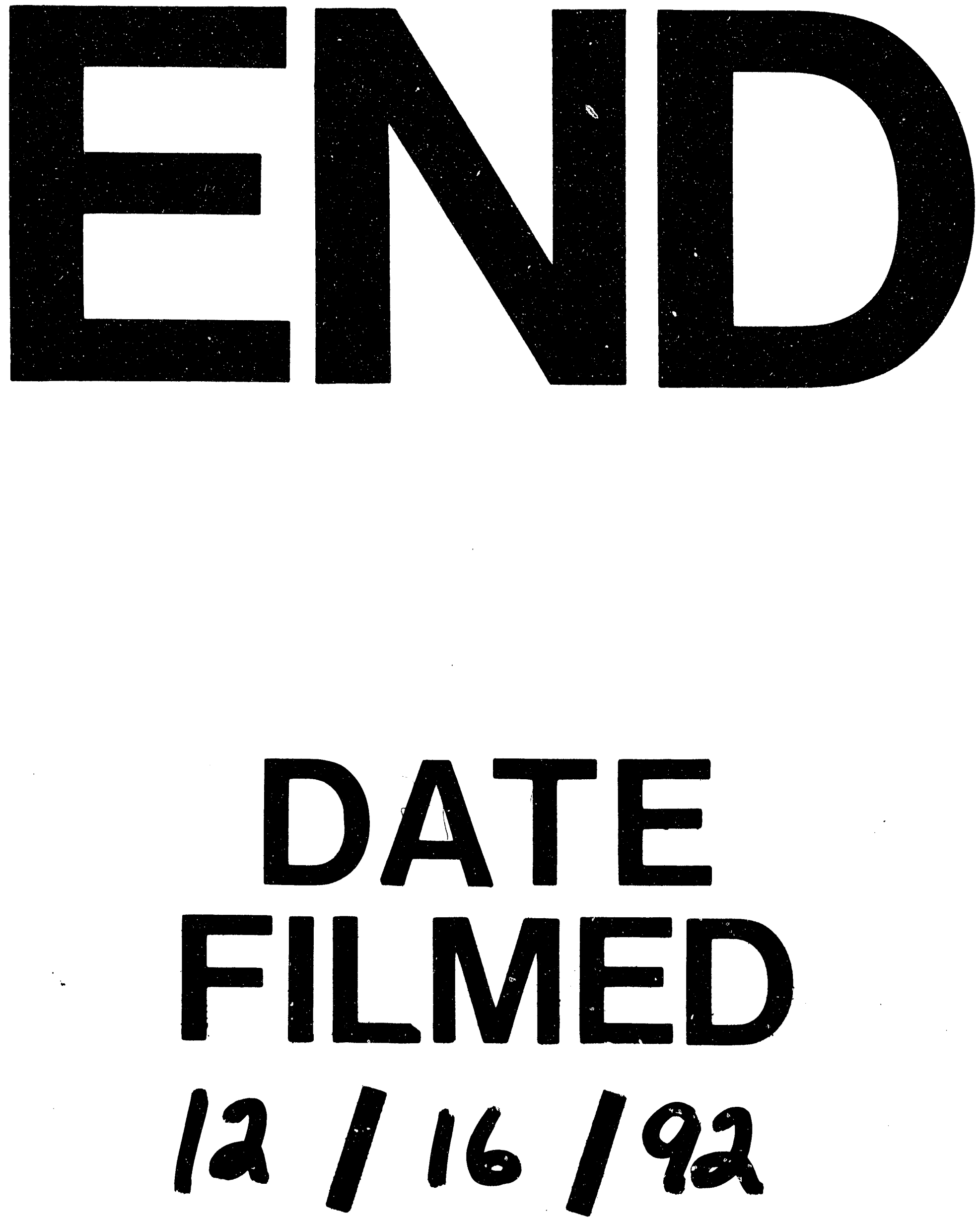
